\title{
GROUND-BASED NEAR-INFRARED OBSERVATIONS OF \\ GLOBAL SOLAR OSCILLATIONS
}

\author{
L. V. DIDKOVSKY \\ Crimean Astrophysical Observatory, Nauchny, Crimea 334413, USSR \\ and \\ V. A. KOTOV \\ Crimean Astrophysical Observatory, Nauchny, Crimea 334413, USSR \\ and \\ Center for Space Science and Astrophysics, Stanford University, \\ Stanford, CA 94305, U.S.A.
}

\begin{abstract}
Near-infrared (0.7-1.0 $\mu \mathrm{m})$ observations of solar brightness oscillations were performed in 1983-1991 at the Crimean Solar Tower with the use of two $(16 \times 16$ and $32 \times 32)$ photodiode arrays. In 1991 new observations of $p$-modes were made simultaneously in two spectral ranges, near 0.7 and $1.0 \mu \mathrm{m}$. The data is analysed to check for the presence of solar variation with the 160 -min period. It is found that the mean relative amplitude for the 160 -min solar irradiance variation at $0.73-1.65 \mu \mathrm{m}$ wavelengths, $\sim 2 \times 10^{-6}$, is much lower than the upper limits set by the ACRIM and IPHIR space experiments.
\end{abstract}

Key words: infrared: stars - Sun: oscillations

\section{Introduction}

The most valuable information about the deep solar interior can be attained by observing long-period oscillations ( $g$-modes) of the Sun. Yet it is widely believed that the most detailed seismic data on the Sun's interior might come entirely from Doppler measurements of $p$-modes. There are some indications however that acoustic frequencies do not fit the standard model of the Sun the with required accuracy (Gough and Toomre, 1991). Measurements of the solar brightness may provide valuable data to improve helioseismic inferences. Of particulart concern is the 160-min periodicity found in the Sun by several groups of observers (Kotov et al., 1983), which so far lacks a reasonable explanation.

Infrared observations (especially those made in atmospheric transparency windows) are to a large extent free from the Earth's atmosphere influences. The remaining errors connected with the atmosphere and instrument can be substantially reduced by the use of differential (center-to-limb) techniques primarily intended for detection of temporal variations of the limb-darkening profile.

The first infrared study of solar global oscillations was made in 1977-1978 by Koutchmy et al. (1980) at $1.65 \mu \mathrm{m}$ wavelength with the use of a linear mechanical monitor of the solar brightness. As a result, the presence of 160 -min oscillations with a mean differential amplitude $\sim 2 \times 10^{-5}$ was firmly established (Kotov et al., 1983). Later this IR monitor was replaced by modern devices based on the use of two photodiode arrays (PA; $16 \times 16$ and $32 \times 32$ pixels). The spectral windows of the new instruments are determined by the spectral sensitivities of the PA's and glass filters. 


\section{Observations}

The PA-1 $(16 \times 16), 3.83 \times 3.85 \mathrm{~mm}$ in size, is illuminated by a $5.9 \mathrm{~mm}$ pin-hole image of the Sun. The brightness signals were collected from 12 pixels corresponding to the $\mathrm{N}, \mathrm{S}, \mathrm{E}$, and $\mathrm{W}$ limbs at heliocentric distances $\mathrm{r} \sim 0.64$ and to the solar disk center (C) (Didkovsky and Kotov, 1986), with spatial resolution $\sim 0.18 D_{\odot}$ and effective wavelength $0.82 \pm 0.13 \mu \mathrm{m}$. The high $\mathrm{S} / \mathrm{N}$ was ensured by a low ratio, $\sim 2 \times 10^{-5}$, of thermal electrons to to photoelectrically gennerated ones. The registration frequency, $\sim 500 \mathrm{~Hz}$, makes it possible to obtain brightness readings from all 5 areas monitored on the Sun's disk every $24 \mathrm{~ms}$; the data were integrated for 1-min intervals and stored.

The analysis consisted of calculation of the differential signals for 4 limb regions on the disk $(\mathrm{i}=\mathrm{N}, \mathrm{S}, \mathrm{E}$ or $\left.\mathrm{W}): d_{i}=\left(I_{c}-I_{i}\right) / I_{c}\right)$, where $I_{c}, I_{i}$ are the mean intensities of the center-disk and limb regions. The averages of the four values of $d_{i}$ were also computed to get "average limb intensity" signal. Due to low spatial resolution this instrument was devoted exclusively to measurements of low-degree global oscillations $(l \lesssim 5)$.

Another, more powerful, device, using a $32 \times 32 \mathrm{PA}$ and a lens to form a solar image on it, was installed at the Solar Tower in 1986 (Didkovsky and Kotov, 1988). The PA is illuminated by a $9-\mathrm{mm}$ image of the Sun and allows us, under $\sim 0.027 D_{\odot}$ resolution, to study oscillations of low and intermediate degree $(l \lesssim 40)$. The spectral passbands correspond to $0.73 \pm 0.10$ and $0.98 \pm 0.15 \mu \mathrm{m}$, with $\sim 38$ seconds integration time. This new instrument records brightness signals from all 1024 pixels and permits an accurate orientation of the PA with respect to solar equator and, accordingly, permits us to obtain the two-dimensional brightness pattern of the photosphere. Thorough tests have been made to determine the degree of array homogeneity and slow temporal trends of pixel sensitivity. The spatial response function has been calculated for both arrays. The slow daily trends, caused primarily by the Earth's atmospheric transparency variations, have been removed by 2 nd order polynomials fits.

\section{Results}

An example of the power spectrum (PS) of residuals (observations minus trend) is shown in Figure 1. It clearly shows the presence of major peaks corresponding to solar $p$-modes within the range 2.5 to $3.4 \mathrm{mHz}$, with average relative amplitudes $4 \times 10^{-4}$. The most interesting point is a good correlation between the frequencies of dominant peaks in Figure 1 with those inferred by Woodard and Hudson (1983) for $p$-modes of low degree from bolometric measurements on board the SMM satellite. The mean spacing we find between major peaks in Figure 2 corresponds to $68 \mu \mathrm{m}$, exactly that of Woodard and Hudson (1983), supporting our interpretation that the signal analyzed is of pure soalr origin.

The use of PA-2 $(32 \times 32)$ has significantly extended our capability to study space-time characteristics of solar oscillations. After removing 10-min running-mean values, the residuals (observations minus "quiet Sun"; see Didkovsky and Kotov, 1988) exhibit 5-min oscillations of solar brightness (Fig. 2). 


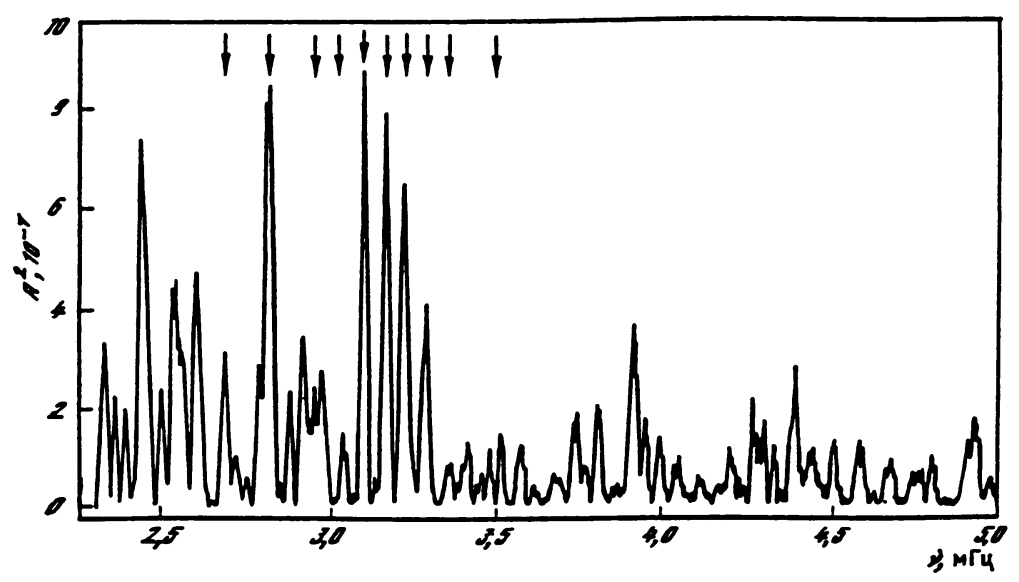

Fig. 1. Power spectrum of the brightness signal $I_{c}(0.82 \mu \mathrm{m}$, August 6,1983$)$. The arrows show 10 frequencies of degree $l=0-2$ inferred from bolometric ACRIM measurements.

In 1991 we observed solar brightness oscillations simultaneously in two spectral windows, $0.73 \pm 0.04$ and $1.00 \pm 0.10 \mu \mathrm{m}$, with the use of the PA-2 device and two sets of glass filters covering separately $\mathrm{N}$ and $\mathrm{S}$ hemispheres of the Sun. The analysis of the observations carried out on 18 and 19 July showed appreciable differences between the power spectra computed (with a spatial filter for the sectorial harmonic degree $l=8, m=0)$ for the $\mathrm{N}(0.73 \mu \mathrm{m})$ and $\mathrm{S}(1.00 \mu \mathrm{m})$ brightness oscillations, with concentration of power near 3.4 and $3.1 \mathrm{mHz}$ respectively (Fig. 3). This study is now in progress, with the main goal of searching for the asymmetry in global oscillations over the solar disk and for plausible differencies between the two spectral ranges.

The photodiode-array data obtained in 1983-1988 at different wavelengths (from 0.7 to $1.0 \mu \mathrm{m}$ ) allow us to check the authenticity of the phase-coherent oscillation with a period of $P_{0}=160.0101 \mathrm{~min}$ (Brookes et al., 1976; Severny et al., 1976). Earlier IR data obtained by Koutchmy et al., 1980 in 1977-1978 at $1.65 \mu \mathrm{m}$ clearly showed the presence of $p_{0}$ oscillation with the mean differential amplitude $\sim 2.5 \times$ $10^{-5}$. This finding was later supported by the 1981-1982 data obtained also at $1.65 \mu \mathrm{m}$ (Kotov et al., 1983). Further evidence in favour of a true solar origin of the 160 -min oscillation was given by careful consideration of all conceivable potentially spurious sources of a potential 160-min periodicity (Didkovsky and Kotov, 1987). The analysis of all data obtained in 1983-1988 with both arrays within the range 0.7-1.0 $\mu$ m supports the conclusion by Koutchmy et al., 1980 about phase stability, in average, of the $P_{0}$ oscillation of the Sun. The average amplitude, however, is found to be several times smaller than that in the previous years, 1977-1982, (a similar decrease of the $P_{0}$-amplitude during a few late years was reported also by Kotov et al., 1992 on the basis of velocity observations).

We now present a superposed-epoch analysis of the total set of brightness mea- 


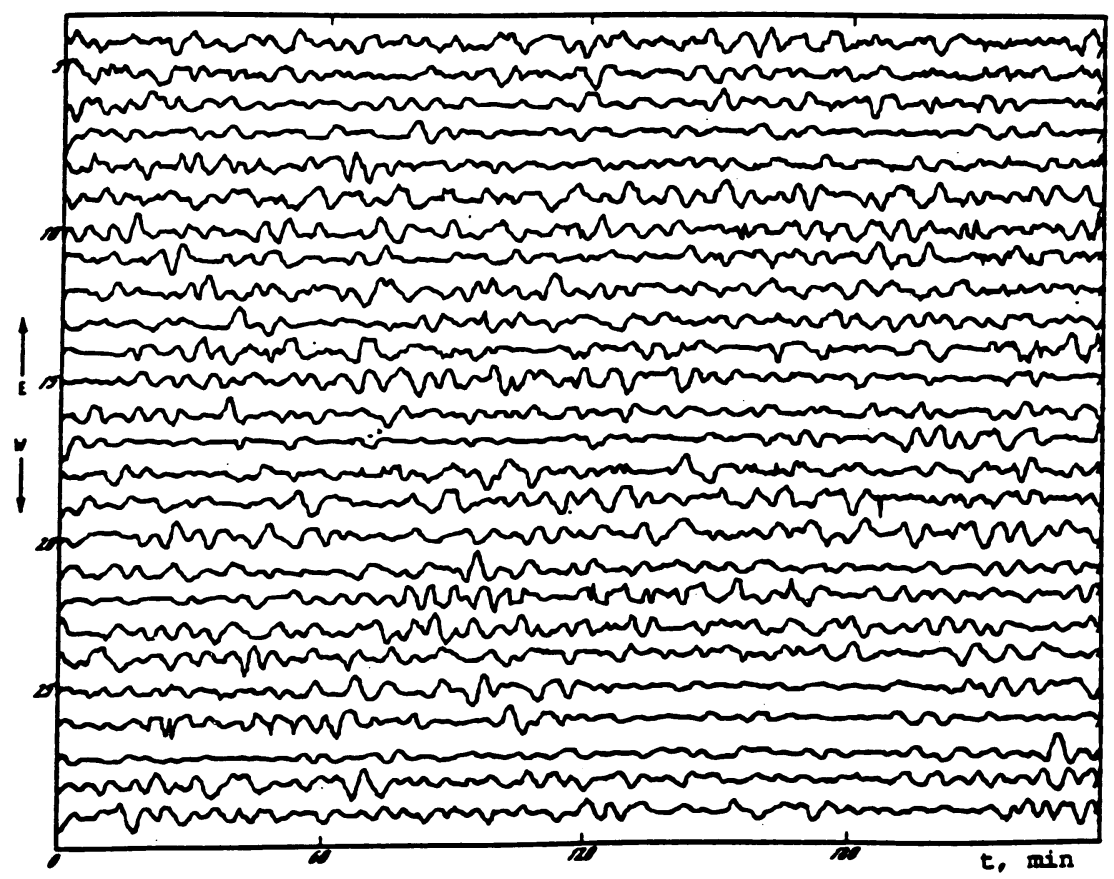

Fig. 2. Two-dimensional time-spatial distribution of 5-min brightness oscillations of the Sun as recorded by pixels 4-29 parallel to the solar equator. Spatial resolution corresponds to $53^{\prime \prime}$; maximum amplitude of brightness oscillations $\sim 0.25 \%$.

surements made in the Crimea from 1976 through 1988. This data is mostly in the near IR [at $0.7-1.0 \mu \mathrm{m}$ - with the use of two PA's, and at $1.65 \mu \mathrm{m}$ - using the IR monitor (Koutchmy et al., 1980)]. In all there are 3102 hours of observations over 565 days (the total number $N$ of 5 -min periods is 37227 ). The resultant brightness curve for the folding period, $P_{0}$ is shown in Figure 4 . The presence of $P_{0}$ periodicity in this 13-year set of observations appears to be highly significant (at about $4 \sigma$ confidence level). The most interesting is a perfect coincidence of the maximum of the near-IR brightness curve with the maximum of flare occurrences on the Sun. The latter curve was obtained by Kotov et al. (1992) from the analysis of $N_{f}=24410$ chromospheric flares of importance $B \geq 1$ observed in 1935-1980.

The average harmonic amplitude of the differential brightness $P_{0}$ variation is $\sim 1.5 \times 10^{-5}$. It corresponds to $\sim 2 \times 10^{-6}$ for an amplitude of solar irradiance changes in the $0.73-1.65 \mu \mathrm{m}$ spectral range (Didkovsky and Kotov, 1987), and is therefore much smaller than the upper limits inferred from bolometric and irradiance measurements performed in 1980 and 1988 on board the SMM and PHOBOS space missions (see, e.g., Woodard and Hudson, 1983). 

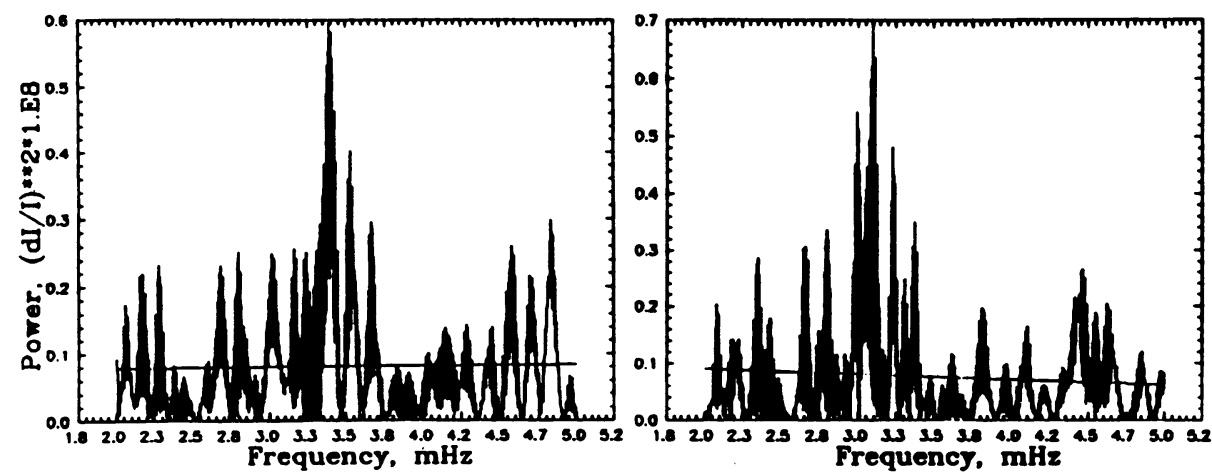

Fig. 3. The power spectra of solar brightness oscillations with spatial filter $l=8, m=0$ for the spectral windows $0.73 \pm 0.10 \mu \mathrm{m}$ (left) and $1.00 \pm 0.04 \mu \mathrm{m}$ (right).
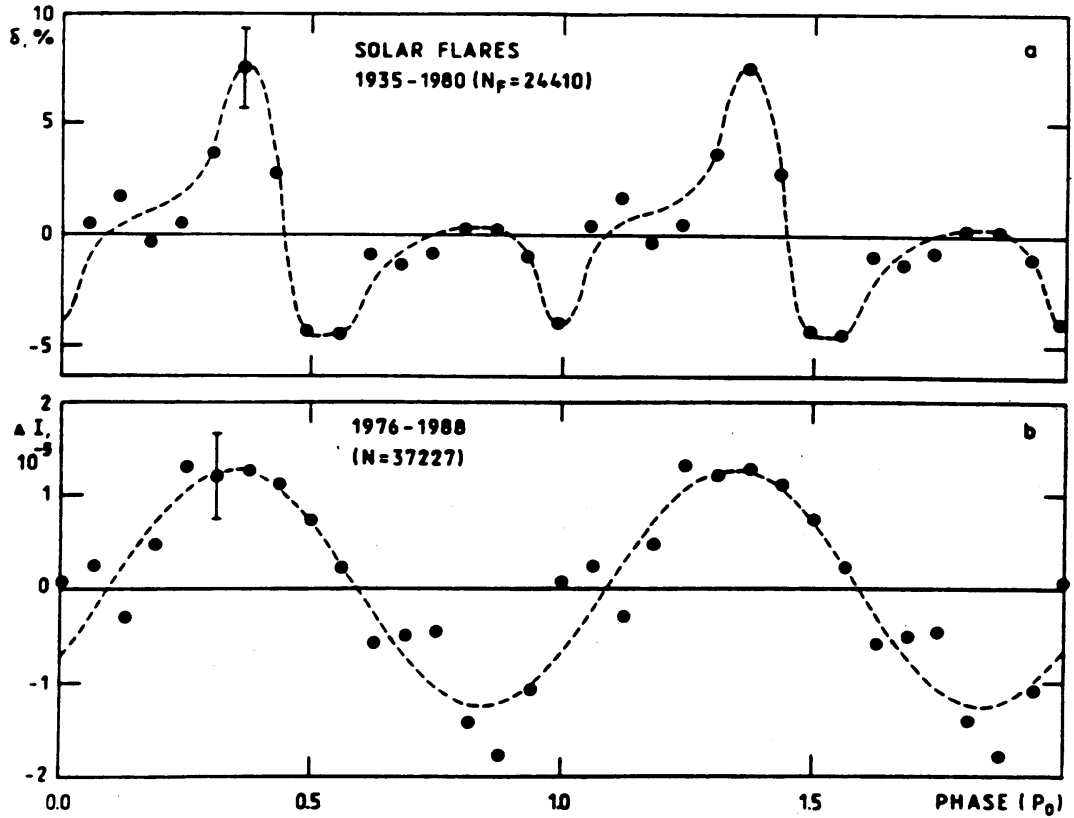

Fig. 4. The average solar brightness $P_{0}$ curve (b), compared with the average profile of frequency modulation of solar flares, 1935-1980, with a period $P_{0}=160.0101 \mathrm{~min}$, (a). 


\section{References}

Brookes, J.R., Isaak, G.R., and van der Raay, H.B.: 1976, Nature 259, 92.

Didkovsky, L.V., and Kotov, V.A.: 1986, Izv. Krym. Astrofiz. Obs. 74, 132.

Didkovsky, L.V., and Kotov, V.A.: 1987, Izv. Krym. Astrofiz. Obs. 76, 119.

Didkovsky, L.V., and Kotov, V.A.: 1988, Izv. Krym. Astrofiz. Obs. 80, 118.

Gough, D., and Toomre, J.: 1991, Ann. Rev. Astron. Astrophys. 29, 627.

Kotov, V.A., Haneychuk, V.I., and Tsap, T.T.: 1992, Crimean J. Astrophys., in press.

Kotov, V.A., Koutchmy, S., and Koutchmy, O.: 1983, Solar Phys. 82, 21.

Koutchmy, S., Koutchmy, O., and Kotov, V.A.: 1980, Astron. Astrophys. 90, 372.

Severny, A.B., Kotov, V.A., and Tsap, T.T.: 1976, Nature 259, 87.

Woodard, M., and Hudson, H.: 1983, Solar Phys. 82, 67. 